\title{
Photoacoustic spectroscopy to determine the optical properties of thin film $4 \mathrm{H}-\mathrm{SiC}$
}

\author{
N.G.C. Astrath ${ }^{\text {a,* }}$, A.C. Bento ${ }^{\text {a }}$, M.L. Baesso ${ }^{a}$, A. Ferreira da Silva ${ }^{b}$, C. Persson ${ }^{c}$ \\ a Departamento de Física, Universidade Estadual de Maringá, 87020-900, Maringá, PR, Brazil \\ ${ }^{\mathrm{b}}$ Instituto de Física, Universidade Federal da Bahia, Campus Univ. de Ondina 40210340 Salvador, BA, Brazil \\ ${ }^{c}$ Department of Materials Science and Engineering, Royal Institute of Technology, SE-100 44 Stockholm, Sweden
}

Received 17 March 2006; received in revised form 29 June 2006; accepted 6 August 2006

Available online 28 September 2006

\begin{abstract}
The optical transitions in a range of $1.5-5.2 \mathrm{eV}$ of n-type 4H-SiC have been investigated experimentally by photoacoustic spectroscopy and theoretically by a full-potential linearized augmented plane wave method. From the absorption spectrum, we found the indirect optical bandgap at $3.2 \mathrm{eV}$ and the direct transitions around $4.5 \mathrm{eV}$ in very good agreement with what has been predicted by theoretical calculations.

(C) 2006 Elsevier B.V. All rights reserved.
\end{abstract}

PACS: 61.82.Fk; 62; 71.23.Cq; 73

Keywords: Silicon carbide; Photoacoustic spectroscopy

\section{Introduction}

$4 \mathrm{H}-\mathrm{SiC}$ is a prominent semiconductor material for highpower, high-temperature, and high-frequency devices with large applicability, such as field effect transistors and ultraviolet detectors $[1,2]$. There is a lack of more information on optical transitions beyond the indirect bandgap energy. Recently the optical and transport properties of the doped $4 \mathrm{H}-\mathrm{SiC}$ have been further investigated [2-4]. In this work, we have investigated the optical properties of $n$-type $4 \mathrm{H}-\mathrm{SiC}$, both experimentally and theoretically. The photoacoustic spectroscopy technique has been used to measure the optical absorption spectrum. The present theoretical study of the electronic structure of $4 \mathrm{H}-\mathrm{SiC}$ is based on the local density approximation (LDA) within the density functional theory, employing the first-principle, fullpotential linearized augmented plane wave method [5]. We have therefore made an estimate of the correction $\Delta_{\mathrm{g}}$ to the bandgap by using a quasi-particle $(\mathrm{QP})$ method proposed by Bechstedt and Del Sole $[6,7]$. Here we present the results and discuss for

\footnotetext{
* Corresponding author.

E-mail address: astrath@dfi.uem.br (N.G.C. Astrath).
}

theoretical calculations, while the readers are called for further details in the cited references and in Ref. [7]. Finally, a detailed discussion about all the transitions is presented.

\section{Experimental procedures}

\subsection{Sample}

The measured sample for the spectroscopy experiments was n-type $4 \mathrm{H}-\mathrm{SiC}$, doped around $7 \times 10^{18} \mathrm{~cm}^{-3}$ with a Si-face, 25$\mu \mathrm{m}$-thick epilayer grown by hot wall chemical vapour deposition [8].

\subsection{Photoacoustic spectroscopy}

In brief, the photoacoustic effect consists of illuminating a given material with a modulated light beam and measuring the subsequent temperature fluctuation induced in the sample resulting from the light absorption, due to nonradiative deexcitation processes within the sample. The intermittent heat is transferred into the sealed gas chamber generating an acoustic signal that can be detected by a microphone [9]. The experimental setup is described in Fig. 1 [10]. The radiation source is 
a 1000 W Xenon Arc lamp (Oriel Corporation 68820). The light passes through a monochromator (Oriel Instruments 77250). Monochromatic light is then diffracted in a narrow band wavelength by a grating monochromator (Oriel 77296), which permits the scanning of a region from 200 to $800 \mathrm{~nm}$. The light comes out through a slit of $3 \mathrm{~mm}$ wide and passes through a mechanical modulator (chopper) (Stanford Research Systems SR540) that contains a holed disk that gyrates in such a stable manner that it generates a reference signal to the detection setup. To eliminate the superior order diffraction, we use band pass filters that cut off second order, and after that the beam is collimated by two quartz lens having focal length $f_{1}=100 \mathrm{~mm}$ and $f_{2}=150 \mathrm{~mm}$. The light goes through a quartz window and reaches the sample inside the chamber. The photoacoustic cell has a sensitive microphone (Brüel e Kjaer, BK 2669) connected to it which transduces the signal and feeds it into a Lock-in amplifier (EG\&G Instrument 5110), which synchronizes the photoacoustic signal with the reference pulse from the chopper. All photoacoustic spectra were performed in the modulation frequency of $20 \mathrm{~Hz}$. System acquisition is attained automatically through a personal computer that controls all instrumentations and also normalizes spectra with fine carbon black. All the experiments were performed at room temperature and by using non-polarized light.

\section{Results}

Fig. 2 shows the average photoacoustic optical absorption spectra of $4 \mathrm{H}-\mathrm{SiC}$ after three runs, which show a band with a peak position of the absorption identified as the optical bandgap around $E_{\mathrm{g}}=3.2 \mathrm{eV}$. This bandgap is originated from the phonon-assisted optical transition. It can also be noted that four other absorption bands are present, a broad band at $2.0 \mathrm{eV}$ and the others around 4.5, 4.6, 5.0 and $5.1 \mathrm{eV}$, characteristics of semiconductors behavior supported by theoretical calculations for the same 4H-SiC $[7,11]$. The absorption band (around the peak position) at $3.2 \mathrm{eV}$ agrees with the value found in the literature [12]. Another peak at about $4.7 \mathrm{eV}$ was observed in some spectra, although it is not predicted in the theoretical cal-

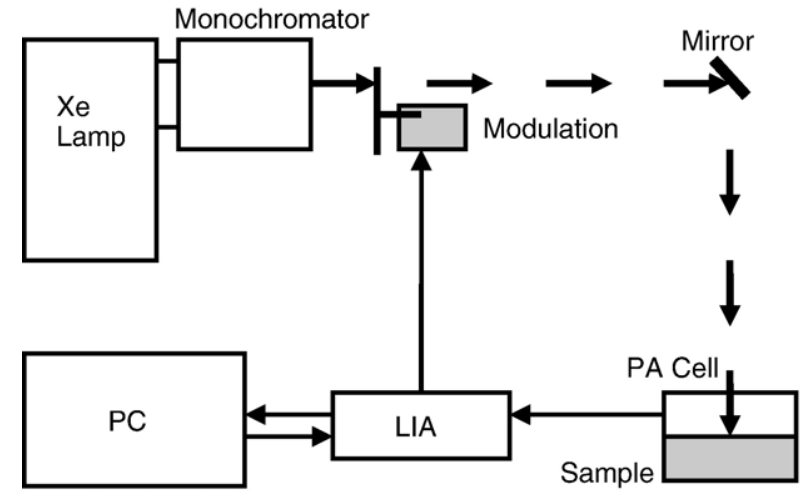

Fig. 1. Home made photoacoustic spectrometer: Schematic diagram. PC: acquisition system; LIA: lock-in amplifier; PA Cell: photoacoustic cell.

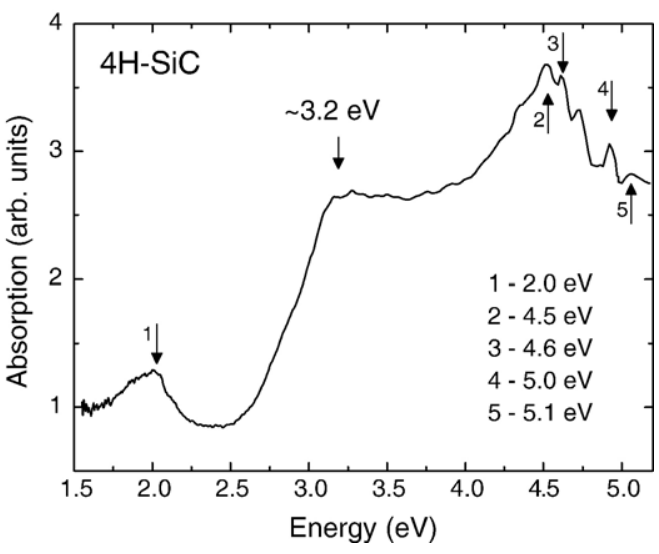

Fig. 2. The absorption spectrum of $4 \mathrm{H}-\mathrm{SiC}$ in arbitrary units, as a function of photon energy.

culations. Thus, as it was not possible to assign its origin, it was not included in our analysis. The origin to this peak may be phonon assisted absorption since the optical phonon energy is about $0.1 \mathrm{eV}$, and both peaks are about $(4.6+0.1) \mathrm{eV}$.

\section{Discussion}

As is well known [9,13-15], the optical absorption measurements is sensitive to doping amount, which means that there are a lot of carriers that are excited in transitions into the conduction band. The bandgap value of about $3.27 \mathrm{eV}$ for intrinsic material at room temperature [12] will be lower in energy due to the screening of the temperature-induced excitations of electrons. For an impurity concentration of $7 \times 10^{18} \mathrm{~cm}^{-3}$ the freeelectron concentration is about $5 \times 10^{17} \mathrm{~cm}^{-3}[16]$ and with this gas of free electrons, the bandgap is reduced by about $40 \mathrm{meV}$ [17], yielding a bandgap of about $3.23 \mathrm{eV}$. This is in good agreement to the measured value presented here $[18,19]$.

The conduction-band minimum of $4 \mathrm{H}-\mathrm{SiC}$ is at the M-point. The second lowest conduction-band minimum is located at the $\mathrm{M}$-point for $4 \mathrm{H}-\mathrm{SiC}$, and consequently in $4 \mathrm{H}-\mathrm{SiC}$, as in $3 \mathrm{C}-\mathrm{SiC}$ [7], there can be direct transitions between the two conductionband minima. The energy difference between the two lowest minima is small in $4 \mathrm{H}-\mathrm{SiC}[\Delta E(\mathrm{M})=0.12 \mathrm{eV}]$, which is close to the experimental value of $\sim 0.14 \mathrm{eV}$, obtained by Im et al. [20] from ballistic electron emission microscopy. This energy difference of the two minima is close to the optical phonon energies of $0.10-0.12 \mathrm{eV}$ [18]. The second band will therefore influence the electronic transport properties at high temperatures and/or when high electric fields are applied. For optical properties, the direct bandgap transition energy is important. The energy difference between the two upper valence bands is about $0.5 \mathrm{eV}$. The lowest energy for direct transitions from the valence band to the conduction band is $\Delta E(\mathrm{M})=4.47 \mathrm{eV}$, and the higher is $\Delta E(\mathrm{M})=4.96 \mathrm{eV}$ calculated with the LDA $+\mathrm{QP}$ model. With the extra $0.12 \mathrm{eV}$ up to the second band, there should be transitions also at about 4.59 and $5.08 \mathrm{eV}$ which is in a very good agreement with the peaks observed in our measurements presented in Fig. 2. The reader should be addressed to Ref. [7] to all detailed calculations concerning to the band 
structure, energy transitions and LDA correction. The direct $\Gamma$ point transition energy in $4 \mathrm{H}-\mathrm{SiC}$ is $\Delta E(\Gamma)=6.31 \mathrm{eV}$.

The absorption band around $2.0 \mathrm{eV}$, which is about $1.2 \mathrm{eV}$ below the conduction band edge, can be identified as $\mathrm{Cr}$ or $\mathrm{V}$ levels presented at the $\mathrm{n}$-doping $4 \mathrm{H}-\mathrm{SiC}$, as pointed out by Achtziger and Witthuhn [21]. Sridhara et al. [22] have observed this band. It has suggested to be due to nitrogen donor levels associated to the transitions with $c_{1}(\mathrm{M} 1)-c_{4}(\mathrm{M} 3)$ [23].

\section{Conclusions}

We have measured the optical transitions of $4 \mathrm{H}-\mathrm{SiC}$, using photoacoustic spectroscopy. The resulting absorption spectrum with different optical transitions in a range of $1.5-5.2 \mathrm{eV}$ is in excellent agreement with the previously theoretical calculations. We have identified the optical transitions from the electronic structure as being transitions at the $\mathrm{X}$ point with the effects of the two conduction band minima around $4.5 \mathrm{eV}$ corresponding to where the direct transition is situated. For the indirect transition, i.e., the bandgap energy, it is found to be $3.2 \mathrm{eV}$.

\section{Acknowledgments}

The authors are grateful to the Brazilian National Research Council (CNPq), Brazilian Network REMAN/CNPq, CAPES, Fundação Araucária, the Swedish Research Council (VR) and the Swedish Foundation for International Cooperation in Research and Higher Education (STINT) for the financial support of this work.

\section{References}

[1] H. Morkoc, S. Strite, G.B. Gao, M.E. Lin, B. Sverdlov, M. Burns, J. Appl. Phys. 76 (1994) 1363.

[2] A. Paskaleva, R.R. Ciechonski, M. Syväjärvi, E. Atanassova, R. Yakimova, J. Appl. Phys. 97 (2005) 124507.
[3] I.G. Ivanov, A. Henry, E. Janzén, Phys. Rev., B 71 (2005) 241201.

[4] J. Pernot, S. Contreras, J. Camassei, J. Appl. Phys. 98 (2005) 023706.

[5] P. Blaha, K. Schwarz, P. Dufek, R. Augustyn, WIEN95, Technical University of Vienna 1995. [Improved and updated UNIX version of the original copyrighted WIEN-code, which was published by P. Blaha, K. Schwarz, P. Sorantin, and S.B. Trickey, in Comput. Phys. Commun. 59 (1990) 399].

[6] F. Bechstedt, R. Del Sole, Phys. Rev., B 38 (1998) 7710.

[7] C. Persson, A. Ferreira da Silva, in: M. Razeghi, M. Henini (Eds.), Optoelectronic Devices, III-Nitrides, Elsevier Advanced Technology, Oxford, 2004, p. 479.

[8] Q. Wahab, A. Ellison, A. Henry, E. Janze'n, C. Hallin, J. Di Persio, R. Martinez, Appl. Phys. Lett. 76 (2000) 2725.

[9] A. Rosencwaig, Photoacoustics and Photoacoustic Spectroscopy, Wiley, New-York, NY, 1980.

[10] A.C. Bento, D.T. Dias, L. Olenka, A.N. Medina, M.L. Baesso, Braz. J. Phys. 32 (2002) 483.

[11] C. Persson, U. Lindefelt, J. Appl. Phys. 82 (1997) 5496.

[12] G.L. Harris, in: G.L. Harris (Ed.), Properties of Silicon Carbide, INSPEC, London, 1995.

[13] C. Donga, X. Lia, D. Niea, L. Xub, Z. Zhangc, Thin Solid Films 461 (2004) 48

[14] A. Mandelis, R. Bleiss, F. Shimura, J. Appl. Phys. 74 (1993) 3431.

[15] J.L. Pichardo, E. Marín, J.J. Alvarado-Gil, J.G. Mendoza-Alvarez, A. Cruz-Orea, I. Delgadillo, G. Torres-Delgado, H. Vargas, Appl. Phys., A 65 (1997) 69.

[16] C. Persson, U. Lindefelt, J. Appl. Phys. 83 (1998) 266.

[17] C. Persson, U. Lindefelt, B.E. Sernelius, Phys. Rev., B 60 (1999) 16479.

[18] I.G. Ivanov, U. Lindefelt, A. Henry, O. Kordina, C. Hallin, A. Aroyo, T. Egilsson, E. Janzén, Phys. Rev., B 58 (1998) 13634.

[19] S.G. Sridhara, S. Bai, O. Shigiltchoff, R.P. Devaty, W.J. Choyke, Mater. Sci. Forum 338 (2000) 567.

[20] H.-J. Im, B. Kaczer, J.P. Pelz, S. Limpijumnong, W.R.L. Lambrecht, W.J. Choyke, J. Electron. Mater. 27 (1998) 345.

[21] N. Achtziger, W. Witthuhn, Appl. Phys. Lett. 71 (1997) 110.

[22] S.G. Sridhara, S. Bai, O. Shigiltchoff, R.P. Devaty, W.J. Choyke, Mater. Sci. Forum 338 (2000) 551.

[23] S. Limpijumnong, W.R.L. Lambrecht, S.N. Rashkeev, B. Segall, Phys. Rev., B 59 (1999) 12890. 